\title{
Finite Element Simulation Research on Medium Plate Multi-Pass Welding Temperature Field
}

\author{
Huawei Liu ${ }^{*}$ and Linxin Niu
}

School of Civil Engineering and Architecture, Huanghuai University, Zhumadian, 463000, China

\begin{abstract}
V-grooved steel plate 30mm in thickness has been taken into consideration to research on the distribution of temperature field for the multi-pass welding of steel plate with intermediate thickness in the whole process of welding and cooling. With the establishment of a reasonable three-dimensional solid model, the utility of ANSYS software for finite element analysis, the usage of birth-death elements and the setting of normal Gauss heat source, steps have been undertaken to achieve the goal that the process of multi-pass welding about steel plate in intermediate thickness is simulated and the law of distribution about welding temperature field is obtained. The research results indicate the following: the temperature in every point of base metal changes constantly when the steel plate $30 \mathrm{~mm}$ in thickness is being welded, the heat input position and position behind heat source are the integral parts which have the highest temperature during the process that welded joints are welded, the temperature gradient in front of welding arc is pretty large as well as that behind welding arc is relatively small. The calculation results are consistent with actual observations providing exactly reliable references for stress field of the welding on steel plates.
\end{abstract}

Keywords: Bending, distortion, matlab, the center point, tilt.

\section{INTRODUCTION}

With the rapid development of the construction steel structure in China and the increasing utility of the high strength steel plates and heavy steel plates, a series of higher skills are required in steel structure welding. But now more importance in research is placed on how much stress, during welding, is produced out of the components in high-temperature welding and how much residual stress is produced in the components, which are to determine their influence on the intensity, and on the reliability and the dimensional accuracy of the components [1]. In the simulation analysis on medium plate welding, some of the difficulties are the establishment of the model, the mesh welding and the selection of the welding heat source directly affecting the accuracy and reliability, the workload and its speed of the computation results [2]. This research is based on the distribution of the temperature field in the multi-pass welding of the steel plate with intermediate thickness in the whole process of welding and cooling, providing exact reliable references to the stress field of welding by utility of finite element method in the multi-pass welding of the medium plates in threedimensional entity.

\section{ANSYS MODEL ANALYSIS ON THE MULTI-PASS WELDING OF THE STEEL PLATE WITH INTERMEDIATE THICKNESS}

\subsection{The Establishment of the ANSYS Model}

Two Q345-grade steel plates with a size of $100 * 100 * 30 \mathrm{~mm}$ are selected for butt welding. According to

*Address correspondence to this author at the School of Civil Engineering and Architecture, Huanghuai University, Zhumadian, 463000, China;

E-mail: bywj010203@126.com the relevant provisions in Welding Handbook for $\mathrm{CO} 2$ arc welding, with the consideration of the possibility in welding and weld groove processing, welding deformation is, as far as possible, to be reduced, welding materials economized, labor productivity proved and cost lowered. By the requirements in Basic Forms and Sizes of the Welding Grooves in Gas Welding, Manual Arc Welding and Gas Arc Welding GB985-88, and with the regulations for multi-layer welding that the maximum thickness of each layer's welding is $5-6 \mathrm{~mm}$, the final realistic simulation model is established as shown in Fig. (1).

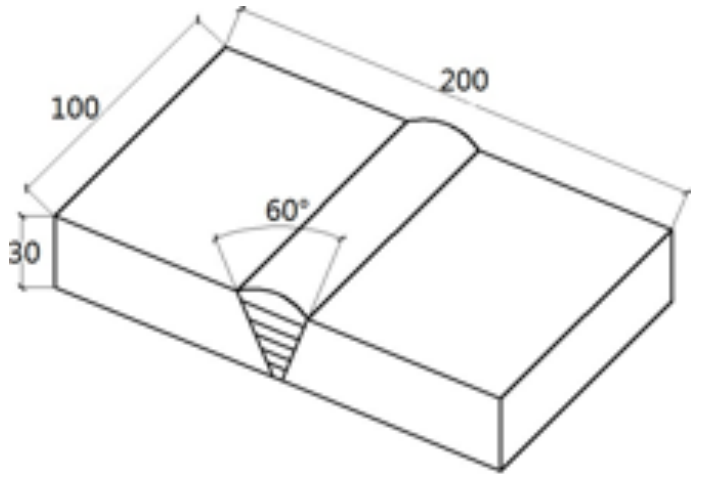

Fig. (1). The model of $30 \mathrm{~mm}$ thick steel plate butt welding.

With the consideration that the welding model networks prolong the computational time and that such characteristics as nonlinear variation of the welding materials are to influence the convergence of the calculation, as well as the required accuracy of the temperature results. ANSYS analysis model is simplified and hypothesized as follows [3]:

1. The initial temperature of the welding structure assembly is $25^{\circ} \mathrm{C}$; 
2. Molten metal liquid flow atmosphere and the chemical reaction inside welding pool are not considered;

3. Welding speed is balanced with the employment of the welding heat source in accordance with the normal Gauss distribution;

4. The heat convection between the welding structure assembly and air is only considered;

5. The differences in the material properties of welding wire materials and those of matrix materials are not considered, while both of them are defined as consistent physical property parameters;

6. The model size is to be relatively small compared with the actual member size;

7. With the consideration of the symmetry of the geometry and load, semi-solid modeling method is to be applied, as shown in Fig. (2).

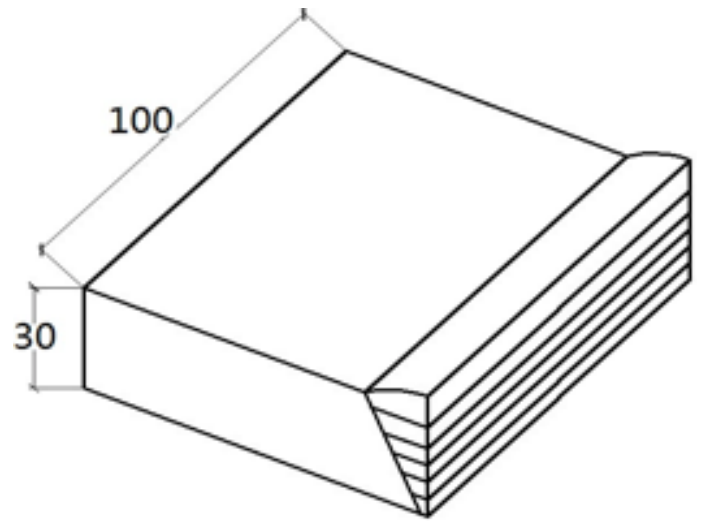

Fig. (2). The model of $30 \mathrm{~mm}$ thick steel plate half an entity.

\subsection{The Selection of the Element Type}

With more than 100 element types with different properties in finite element analysis software ANSYS, the selection of a certain element type requires the analysis of the physical property (including the material types, model geometry, mechanical property and load type etc). What can be applied to thermal analysis is mainly as follows: the twodimensional element PLANE35, PLANE55, PLANE77 and PLANE78, and the three-dimensional element SOLID70 and SOLID90 [4].

The object of this research being the multi-pass welding simulation of the medium plates, first requires the temperature field distribution of the direction within the steel plates, thus utilizing three-dimensional solid elements. The solid elements should have the ability to finish the heat transfer analysis, with the elements being able to carry out the nonlinear transient analysis. Arcs with irregular shapes are included in the $30 \mathrm{~mm}$ steel plate welding model. Element SOLID90 is a thermal analysis element which is provided with an intermediate node among the 20 nodes, each of them bearing the temperature degree of freedom as well as the temperature function, which can be applied to the causes of modes and can configure the steady-state analysis or the transient analysis. So the element SOLID90 is selected for its comparative appropriateness.

\subsection{Meshing}

Meshing methods of ANSYS are categorized into free meshing and regular mapping meshing [5], the former being available for the models in irregular shapes or the transitional region requiring the transition from the fine grids to the sparse grids.

In the computation of ANSYS, the computational accuracy of the element meshes is in proportion to their fineness while the time efficiency is out of proportion to that. During the welding of medium plates, great temperature gradient emerges in welding seams and the region towards them, with the reason that element meshing in those regions requires far more fineness while, conversely, the element meshing away from welding seam regions is relatively sparse. With the overall consideration of the accuracy and time efficiency, the element mesh size of the welding seam regions in the simulation computation herein is controlled in no more than $2 \mathrm{~mm}$, with the application of comparatively larger size in other regions. Fig. (3) presents the meshing of the simulated $30 \mathrm{~mm}$ model of the steel plate welding.

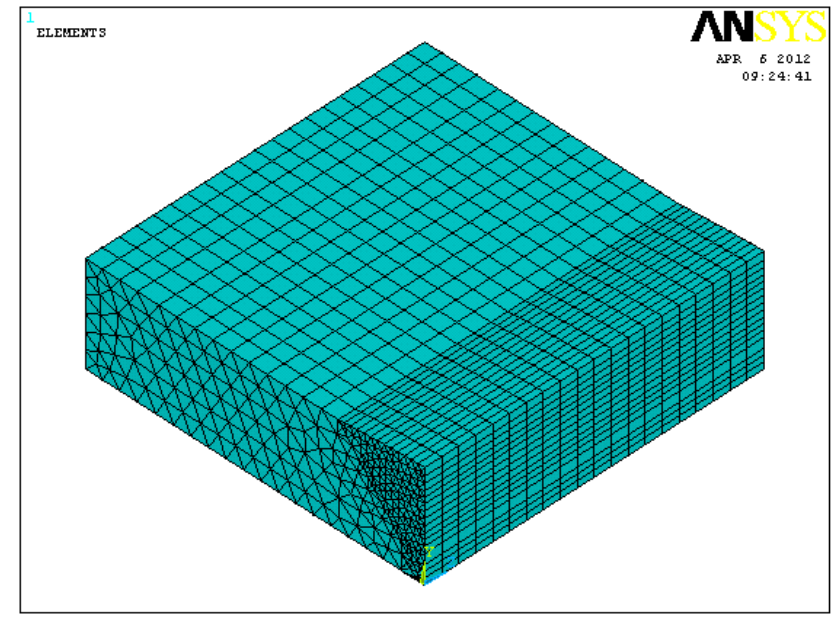

The number of key points: 54

The number of nodes: 29896

The number of elements: 9460

Fig. (3). Finite element mesh model.

\subsection{The Setting up of the Process Parameter and the Material Property}

This research simulates the semi-automatic carbon dioxide protecting the welding of the steel plates $30 \mathrm{~mm}$ in thickness, the selection of welding parameter mainly including the thickness of the steel plates, the diameter of the wires, the speed of the welding, the voltage in welding, the current in welding and the effective radius of the welding heat resource, as presented in Table $\mathbf{1}$.

In setting up the property parameter of the welding materials during the ANSYS simulation analysis, the thermal physical property parameter is set up at the typical temperature and the performance parameter database of the thermophysical materials is established, with the utility of interpolation and extrapolation in defining the parameter when the temperature is not presented. In order to solve the problem that the convergence of the ANSYS simulation 
analysis is difficult, the parameter of the welding materials in high temperature is processed as shown in Table 2.

Table 1. $30 \mathrm{~mm}$ thick steel plate welding parameters.

\begin{tabular}{|l|c|}
\hline Type of the Steels & Q345 \\
\hline Speed of Welding & $5 \mathrm{~mm} / \mathrm{s}$ \\
\hline The Heat Efficiency & 0.75 \\
\hline Voltage in Welding & $35 \mathrm{~V}$ \\
\hline Current in Welding & $600 \mathrm{~A}$ \\
\hline Effective Radius of the Welding Heat Resource & $30 \mathrm{~mm}$ \\
\hline Thickness of the Steel Plates & $30 \mathrm{~mm}$ \\
\hline
\end{tabular}

\subsection{The Determination of the Heat Source Model}

The selection of the welding heat source influences the accuracy of the welding temperature field and the stress field simulation. Considering the accuracy and the normal completion of the simulation, the research herein loads the heat source model in the distribution of the normal Gauss function onto every element of the welding seams, in the form of heat generation rate. During the welding of the electronic arc, high energy beam and flame, the model employs the surface heat source model whose heat flux is in normal Gauss distribution to simulate the welding temperature field under that condition, considering that the comparative shallowness of the model's penetration, as well as the unevenness of the heat distribution in heating spots, distributes more heat to the core region of the heat source but less to the region far away from the core.

The power density formula of the heat source in normal Gauss distribution is:

$q(r)=q_{m} e^{-C r^{2}}$

In formula (1), $q(r)$ : the surface heat flux in radius (r);

$q_{m}$ : the maximum heat flux in the core of the heat source;

$C$ : the heat flux concentration coefficient;

$r:$ the radial distance from the points to the core of the heat source.

\subsection{The Filling of Welding Seams and the Loading of Moving Heat Sources}

For the existence of the grooves between the thick steel plates in welding, which requires the filling of the wires, the "birth and death" element technology is employed to fill the welding seams. Every weld groove element is killed in the first place, and then the APDL language of ANSYS is utilized to compile the subprogram, read successively the center coordinates of the required loading element, activate the elements in that region and apply the loading value to every activated element, and afterwards, the loop statement is employed to finish the piecewise activating and loading of the weld groove elements. The specific approaches are: the length (L) of the welding seam that is cut into pieces along the direction of welding, the element activated piecewise, the rear point employed as the heat source center and Gauss distribution heat source loaded to carry out the computation, with the computational time of that piece being $L /(N v)$ and the computation of that piece being defined as a load step; before computing the following load step, the normal Gauss heat flux by the previous one requires elimination and the loading computational temperature value by the previous load step is employed as the initial temperature of the loading calculation in the following one. Such loop computation can simulate the moving of the heat source and finish the filling of the welding seams and the simulated computation of the transient temperature field of welding.

\subsection{The Result Analysis of the Temperature Field in Multi-pass Welding of the Steel Plates}

The post-processing function of ANSYS and the general post processor POST1 are utilized to carry out the result analysis of welding and the image display. The nephogram of the temperature field in the welding of steel plates $30 \mathrm{~mm}$ in thickness is shown as follows in Figs. (4-9).

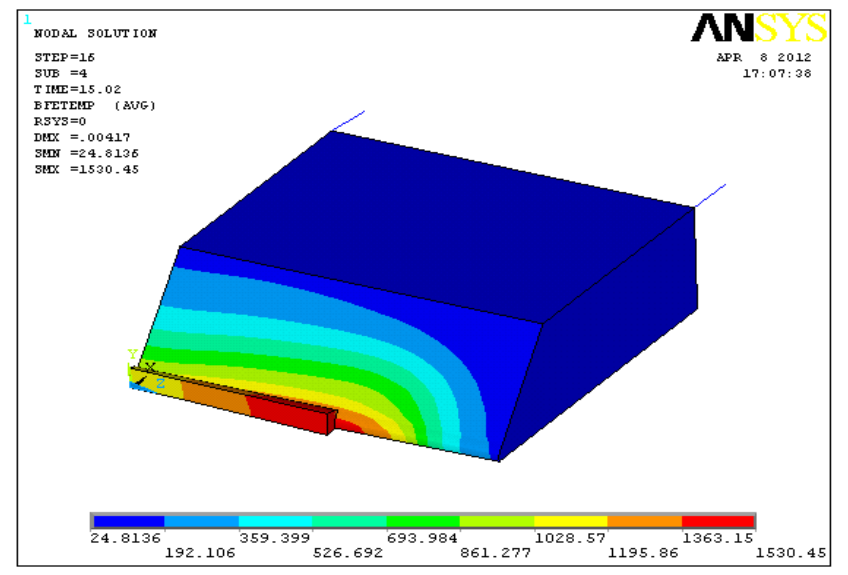

Fig. (4). The first line of weld welding temperature field distribution.

Table 2. Welding performance parameters of material physical properties table.

\begin{tabular}{|c|c|c|c|c|c|c|}
\hline $\mathbf{T}\left({ }^{\circ} \mathbf{C}\right)$ & 20 & 300 & 700 & 1000 & 1500 & 2000 \\
\hline Thermal Productivity $\lambda\left(W / m \mathrm{~g}^{\circ} \mathrm{C}\right)$ & 39 & 33.5 & 26 & 29 & 33 & 33 \\
\hline Density $\rho\left(\mathrm{Kg} / \mathrm{m}^{3}\right)$ & 7800 & 7700 & 7650 & 7600 & 6900 & 6900 \\
\hline Specific Heat Capacity $C\left(J / K g g^{\circ} C\right)$ & 380 & 585 & 920 & 1370 & 2800 & 2000 \\
\hline Heat Transfer Coefficient $\beta\left(W / m \mathrm{~g}^{\circ} \mathrm{C}\right)$ & 100 & 550 & 1000 & 2500 & 3000 & 3200 \\
\hline
\end{tabular}




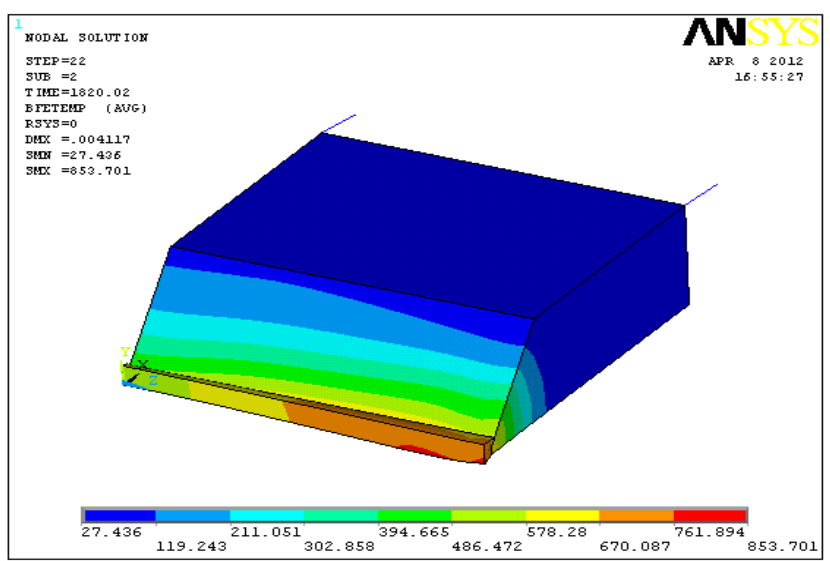

Fig. (5). The first line of weld temperature field distribution of the cooling process.

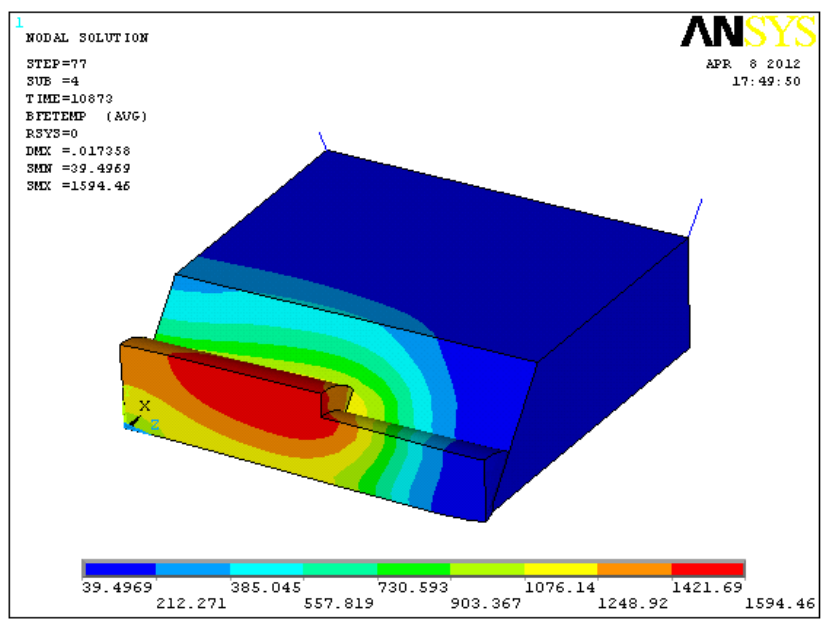

Fig. (6). The fourth weld welding temperature field distribution.

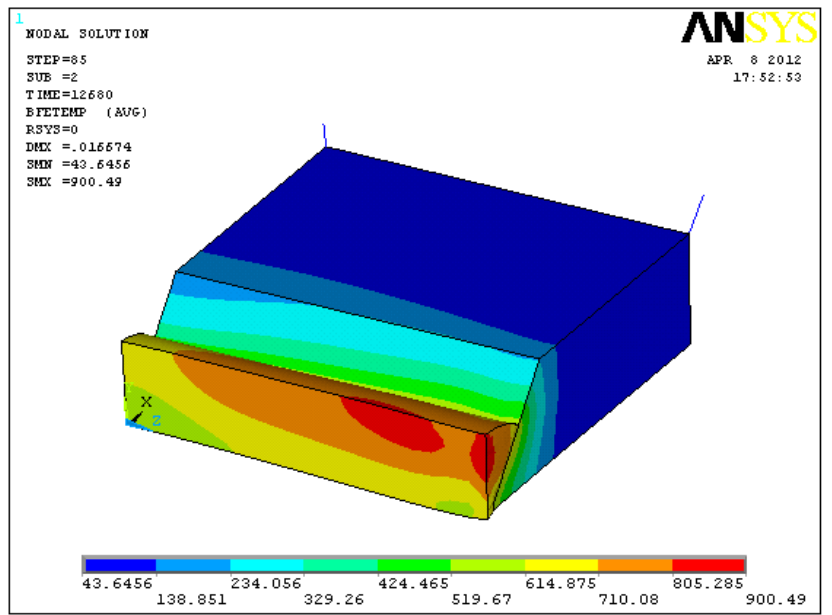

Fig. (7). The fourth weld temperature field distribution of the cooling process.

\subsection{Analysis on the Computation Result}

According to the previous temperature field distribution nephograms, with the continuously moving heat source, temperature in every point of the base metals, in the steel plates with $30 \mathrm{~mm}$ thickness is constantly varying during their welding. In the beginning of every welding seam, the relatively unstable temperature urges the constantly roaring augment of the temperature in base metals. In the welding of every seam, the position where the heat source is inputted and its backside are the regions with the highest temperature while, with the ceaselessly inputting of the heat source, the seams at the backside of the heat source cool themselves being at a slow speed [6-8]. When, therefore, the highest temperature arises at the rear of the heat source, what is observed is that the temperature gradient in the front of the welding arc is much higher than that at the backside. A cooling process emerges after the welding of each seam, during which the temperature in the base metal drops rapidly, and the isotherm range enlarges continuously. Before the commencement of the next seam, the base metal does not cool itself to room temperature, the heat being as the initial heat in the commencement of the next seam. Eventually with the welding being at an end, temperature of the whole weldment drops bit by bit to room temperature; this, however, takes a relatively long time.

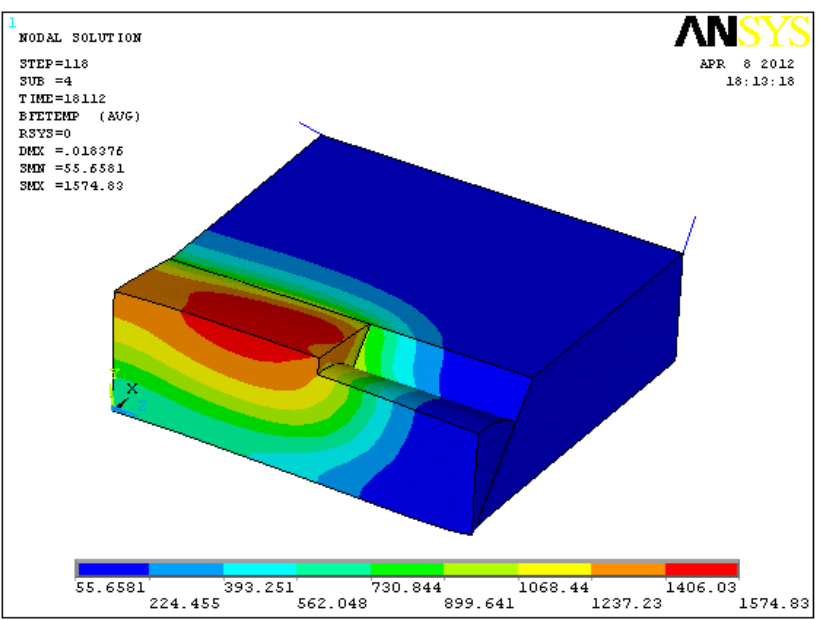

Fig. (8). Sixth welding seam welding temperature field distribution.

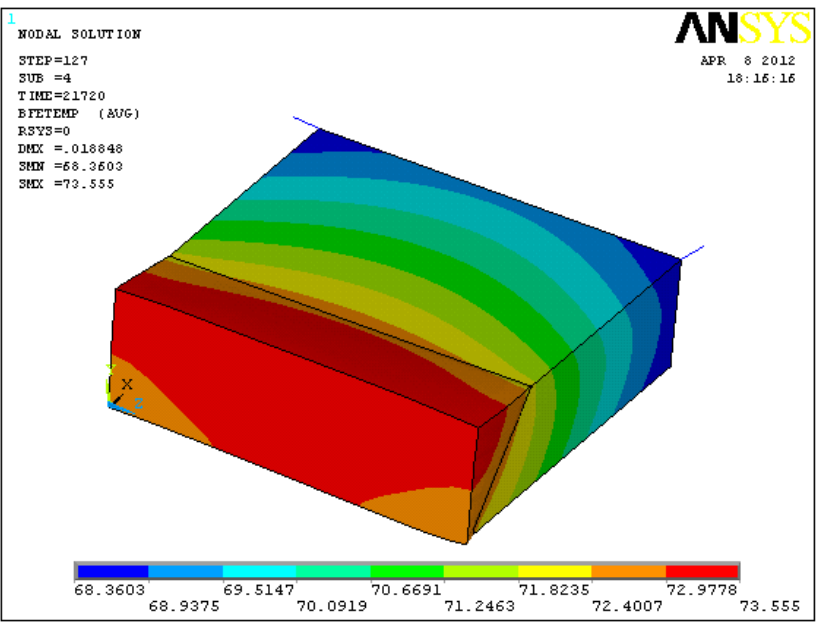

Fig. (9). The sixth weld welding temperature field distribution.

\section{CONCLUSION}

1. The research herein proceeds in the finite element analysis simulation in welding of three-dimensional solid steel plates; 
2. By simulation analysis, the acquired temperature field distribution during the welding of the steel plates is approximately in accordance with the state known in reality;

3. The computation result of the temperature field can be applied to the welding stress analysis and research afterwards, laying a solid foundation in the welding stress research;

4. The finite element simulation program of this research requires further amelioration, improving computational efficiency, providing a foothold for further quantitative analysis of the welding temperature field.

\section{CONFLICT OF INTEREST}

The authors confirm that this article content has no conflict of interest.

\section{ACKNOWLEDGEMENTS}

Declared none.

\section{REFERENCES}

[1] W. Li, "The development on new steel material and the weldability and welding materials in our country's," Machinist Metal Forming, 2005

[2] C. Chen, J. Wang, and H. Yang, "Analysis and calculation of nonlinear welding heat conduction by finite element method," Transactions of the China Welding Institution, vol. 3, no. I39-148, 1983.

[3] Y. Gao, and X. He, "Simulation of Welding Based on ANSYS Unit Birth and Death Technique," Heat Treatment Technology and Equipment, vol.10, 2010.

[4] J. Tan, "Use ANSYS6.0 for finite element analysis," Beijing, Peking University Press, 2002.

[5] S. Tadeusz, Y. Nakasone, and S. Yoshimoto, "Engineering analysis with ANSYS software," Butterworth-Heinemann, 2011.

[6] M. O. Xi-ping, "Simulation and analysis of acoustics transducers using the ANSYS software," Technical Acoustics, vol. 6, 2007.

[7] D. Wang, L. Sun, F. Shan, and H. Xu, "Application of ANSYS Software in Solution of Contact Problem of Bearing," Bearing, vol.9, no.2, pp. 1-4, 2002.

[8] A. G. Arani, R. Rahmani, and A. Arefmanesh, "Elastic buckling analysis of single-walled carbon nanotube under combined loading by using the ANSYS software," Physica E: Low-dimensional Systems and Nanostructures, vol. 40, no.7, pp. 2390-2395, 2008

(C) Liu and Niu; Licensee Bentham Open.

This is an open access article licensed under the terms of the (https://creativecommons.org/licenses/by/4.0/legalcode), which permits unrestricted, non-commercial use, distribution and reproduction in any medium, provided the work is properly cited. 